\title{
Sistema de Aquisição de Dados de Extensometria Aplicado a um Tambor Descascador de Toras de Madeira
}

\author{
Bruno Ferreira de Jesus Junior*, Alan Kardek Rêgo Segundo*
*Departamento de Engenharia de Controle e Automação, Escola de Minas, Universidade Federal de Ouro Preto, campus Morro do Cruzeiro, s/n, Bauxita, Ouro Preto-MG, 35400-000 (e-mail: bruno.junior@aluno.ufop.edu.br, alankardek@ufop.edu.br).

\begin{abstract}
This work presents the development of an extensometer data acquisition system, applied to a wood debarking drum, aiming at the structural integrity analysis of the equipment. The debarking drum operates in a rotating motion to debar wood logs, which are thrown into the structure where contact with the cracked wall occurs. The experiment consists of installing strain gauges in critical areas of the drum structure and performing data acquisition through a system based on the ESP32 microcontroller, aiming to study the structural integrity of the equipment. Data transmission is performed via wireless communication, which facilitates the installation of strain gauges in the drum structure. Based on the results presented, it is possible to evaluate the impacts suffered by the equipment during its operating cycle.
\end{abstract}

Resumo: Este trabalho apresenta o desenvolvimento de um sistema de aquisição de dados de
extensometria, aplicados a um tambor descascador de toras de madeira, visando a análise de integridade
estrutural do equipamento. O tambor descascador opera com movimento de rotação para descascar toras
de madeira, que são lançadas dentro da estrutura onde ocorre o contato com a parede com fendas. O
experimento consiste em instalar extensômetros em áreas críticas da estrutura do tambor e realizar a
aquisição de dados por meio de um sistema baseado no microcontrolador ESP32, visando o estudo da
integridade estrutural do equipamento. A transmissão dos dados é realizada por meio de comunicação sem
fio, o que facilita a instalação dos extensômetros na estrutura do tambor. Com base nos resultados
apresentados, pode-se avaliar os impactos sofridos pelo equipamento durante o seu ciclo de funcionamento.

Keywords: Extensometer; Data Acquisition; ESP32; Arduino; Strain Gage; Sistema Embarcado.

Palavras-chaves: Extensometria; Aquisição de Dados; ESP32; Arduino; Strain Gage; Embedded System.

\section{INTRODUÇÃO}

A aquisição de dados (DAQ) é o processo de medição de um fenômeno elétrico ou físico, como tensão, corrente, temperatura, pressão ou som, com o uso de um computador. Um sistema DAQ é formado por sensores, hardware de aquisição e medição de dados e um computador com software programável. Em comparação com os sistemas tradicionais de medição, os sistemas DAQ baseados em PC exploram a capacidade de processamento, produtividade, sistemas de visualização e recursos de conectividades dos computadores padrão da indústria. Com isso, tem-se uma solução de medição mais poderosa, flexível e de melhor custo-benefício (NI, 2019).

O sensor de deformação é uma das ferramentas mais importantes na técnica de medição elétrica aplicada à medição de grandezas mecânicas. Tecnicamente, "deformação" pode ser causada por tensão e por compressão, diferenciados por um sinal positivo ou negativo. Portanto, os sensores de deformação são utilizados para captar expansão e contração (Omega, 2017).
O sistema de aquisição de dados de extensometria pode auxiliar nos estudos de integridade estrutural, validando estudos com dados coletados em campo.

Neste trabalho, pretende-se apresentar o desenvolvimento e a aplicação de um sistema baseado em microcontrolador para coleta de dados de extensômetros instalados na estrutura de um tambor descascador de toras de madeira, com finalidade de estudos de integridade estrutural.

O tambor descascador tem como função descascar toras de madeira. Ele faz parte da primeira etapa do trabalho com a madeira após a extração, deixando-a preparada para as próximas etapas do processo de fabricação do papel.

\section{TEORIA}

\subsection{Extensometria}

Extensômetros (ou em inglês, strain gauges) são sensores usados para medir deformações mecânicas em corpos de prova. A medida é realizada colando-se um extensômetro no 
local de interesse e medindo-se a variação da sua resistência elétrica, que é função da sua deformação mecânica. Para isso, é necessário transformar a variação da resistência elétrica em variação de diferença de potencial elétrico e, posteriormente, amplificar este sinal antes de realizar a leitura. Dessa forma, deformações em várias partes de uma estrutura real sob condições de serviço podem ser medidas com boa precisão sem que a estrutura seja destruída. Assim, pode-se realizar uma análise quantitativa da distribuição da deformação sob condições reais de operação (Andolfato, 2004; Silva, 2019).

Para exemplificar o princípio de funcionamento de um extensômetro, na Figura 1, apresenta-se um desenho representativo de um fio e sua seção transversal, mostrando-se a deformação de um fio tracionado.

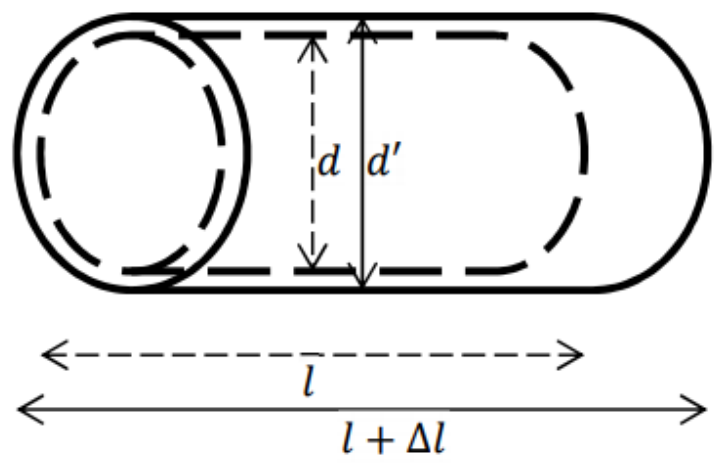

Fig. 1: Deformação em um fio tracionado.

A resistência elétrica dos materiais metálicos se altera diante de uma deformação mecânica. Na Figura 1, por exemplo, o fio metálico sem sofrer nenhuma deformação, i. e., de comprimento $l$ e diâmetro $d$, apresenta resistência elétrica $R$ de acordo com a $2^{\text {a }}$ lei de Ohm:

$$
R=\rho \frac{l}{A}
$$

em que $l$ é o comprimento do fio (m), $A$ é a área da seção transversal do fio $\left(\mathrm{m}^{2}\right), \rho$ é a resistividade do material metálico $(\Omega \cdot \mathrm{m})$ e $d$ é o diâmetro do fio $(\mathrm{m})$.

Caso o material sofra alguma deformação no comprimento $(\Delta l)$ ou no diâmetro $(\Delta d)$, e considerando-se a área da seção do fio condutor $A=4^{-1} \pi d^{2}$, a sua resistência sofre uma alteração $\Delta R$, ou seja:

$$
(R+\Delta R)=4 \rho \frac{l+\Delta l}{\pi(d+\Delta d)^{2}} .
$$

No caso de um exensômetro típico, tem-se um formato de grade constituído por um fio percorrendo em ziguezague o caminho entre os seus dois terminais. Dessa forma, torna-se possível mensurar os esforços submetidos a um corpo de prova por meio da medição da variação da resistência elétrica do extensômetro. O formato de um extensômetro comercial é apresentado na Figura 2, juntamente com a base onde ele é fixado.

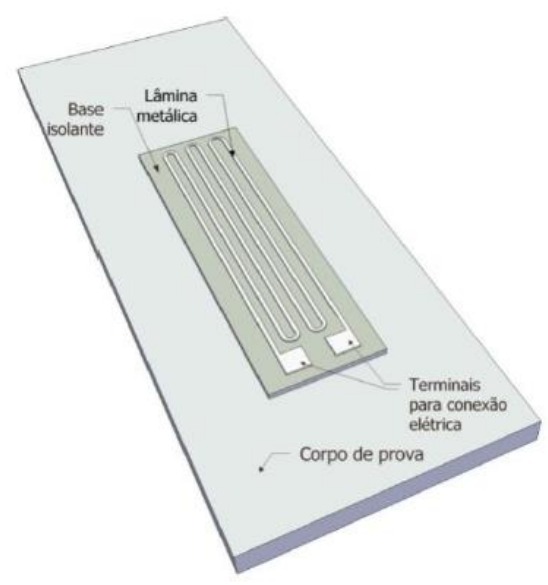

Fig. 2: Diagrama esquemático de um extensômetro de fita metálica, colado sobre um corpo de prova.

Fonte: Guadagnini, 2015.

\subsection{Circuito de medição}

Em extensiometria, utiliza-se o circuito de ponte de Wheastone para transformar a variação da resistência elétrica do(s) extensômetro(s) em variação de diferença de potencial, apresentado na Figura 3.

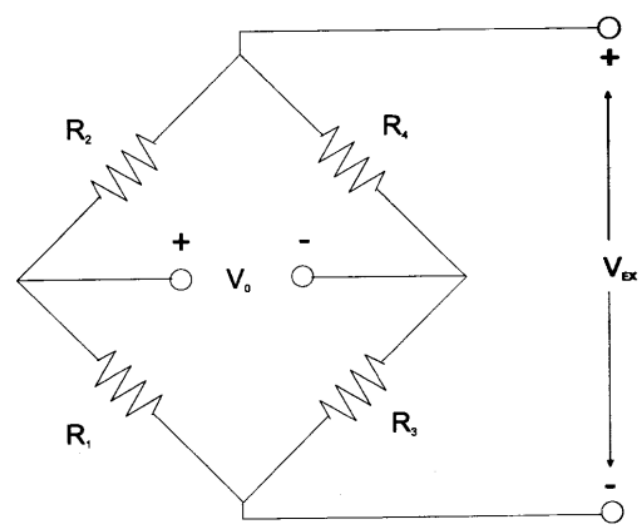

Fig. 3: Circuito elétrico da ponte de Wheastone.

De acordo com a Figura 3, aplicando-se uma tensão de entrada $V_{E X}$ no circuito, tem-se uma tensão de saída $V_{o}$ de acordo com:

$$
V_{o}=V_{e x}\left[\frac{R_{1}}{\left(R_{1}+R_{2}\right)}-\frac{R_{3}}{\left(R_{3}+R_{4}\right)}\right]
$$

em que $R_{1}, R_{2}, R_{3}$ e $R_{4}$ são as resistências da ponte de Wheastone $(\Omega)$.

Caso todos os resistores tenham o mesmo valor, a tensão de saída $V_{o}$ é igual a zero, logo, o circuito está balanceado. Qualquer desbalanceamento gera um sinal de tensão de saída diferente de zero.

Para a aplicação no tambor descascador foram medidas deformações uniaxiais em meia ponte, para medir deformação em uma direção. O primeiro extensômetro é responsável pela medida da deformação na estrutura e o outro é utilizado como uma resistência variável de acordo com a temperatura, contrapondo esse efeito na resistência no primeiro 
extensômetro; e os outros dois são resistências fixas que completam a ponte Wheastone, como apresentado na Figura 4.

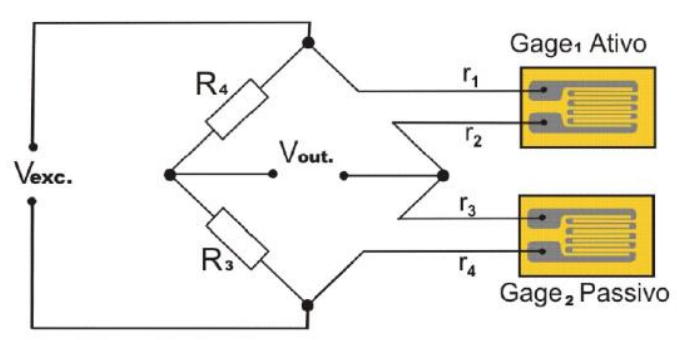

Fig. 4: Diagrama esquemático do sistema em meia ponte. Fonte: Paulino, 2011.

\section{MATERIAIS E MÉTODOS}

Este trabalho foi desenvolvido em uma empresa fabricante de papel e celulose. Para a fabricação do sistema de aquisição de dados foram utilizados dois microcontroladores ESP32, quatro HX711 (Conversor analógico-digital de 24 bits para balanças de pesagem), oito extensômetros, oito resistores de $120 \Omega$ e quatro trimpots de $100 \Omega$. Com uma frequência de aquisição de aproximadamente $40 \mathrm{~Hz}$, o HX711 é um circuito que atende a esta aplicação.

\subsection{O ESP32}

O ESP32 é um chip que contêm Wi-Fi e Bluetooth de 2,4 GHz, projetado com o sensor de ultrabaixa potência da TSMC. Tendo em vista o tamanho reduzido da placa, boa capacidade de processamento e sua possibilidade de trabalhar com o envio de dados sem fio, o ESP32 foi selecionado como boa alternativa para aplicação dentro do sistema de aquisição.

Com a IDE Arduino como opção de compilar e carregar programas, o ESP32 se torna uma ferramenta simples e habitual para o desenvolvimento. A placa de desenvolvimento utilizada no sistema de aquisição de dados, baseada em ESP32, é a ESP32 Thing da Sparkfun (Figura 5), que realiza a coleta e o envio dos dados de extensometria.

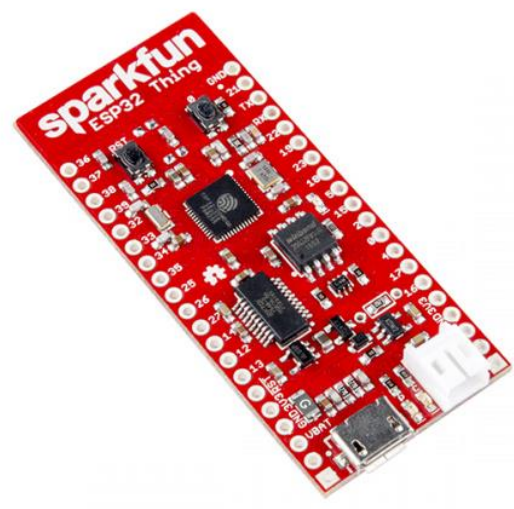

Fig. 5: Placa ESP32 Thing Sparkfun.

Fonte: Sparkfun, 2019.

\subsection{Conversor analógico-digital HX711}

O circuito integrado HX711, representado na Figura 6, é um conversor analógico-digital (ADC) de 24 bits de resolução. Ele pode ser ligado diretamente a um sensor em ponte, sendo projetado para uso em balanças de pesagem (Semiconductor, 2011).

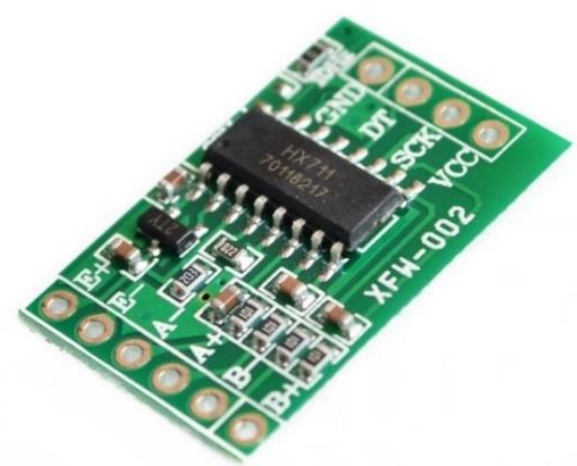

Fig. 6: Placa HX711.

Fonte: Semiconductor, 2011.

O CI HX711 recebe o sinal analógico de saída da ponte Wheastone, amplifica-o, com ganho de 64 vezes ou 128 vezes, e o converte para um sinal digital de 24 bits, que é enviado ao ESP32.

Esse dispositivo foi selecionado pela sua facilidade de utilização, pois já possui as entradas indicadas e os filtros necessários para diminuição de ruídos.

\subsection{Método de calibração}

A calibração do sistema foi realizada por meio de um teste de bancada utilizando uma haste de aço. Uma de suas extremidades foi fixada em uma mesa, e a outra foi submetida a uma força de $2,18 \mathrm{~N}$ em diferentes posições, de forma progressiva (quatro pontos entre as duas extremidades), como mostrado na Figura 7.

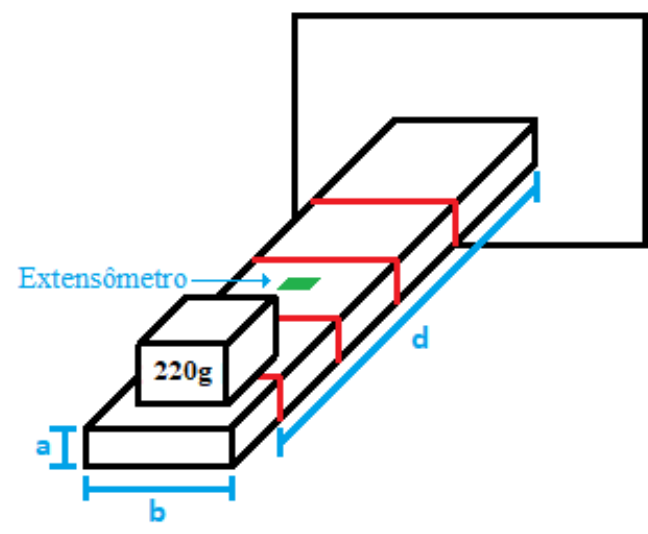

Fig. 7: Esboço dos pontos de calibração. 
Para se obter a curva de calibração, os resultados da conversão analógico-digital (ADC) do sinal do extensômetro foram correlacionados com a tensão mecânica (MPa) aplicada ao sistema, em cada ponto, por meio de regressão linear.

A tensão mecânica $\sigma$, em MPa, foi calculada em cada ponto de calibração como

$$
\sigma=\frac{M c}{I}
$$

em que $M$ é o momento (N.mm), $c$ é a distância da linha neutra até a linha externa, ou seja, metade da largura da haste $(\mathrm{mm})$ e $I$ é o momento de inércia da secção reta transversal do corpo de prova $\left(\mathrm{mm}^{4}\right)$. $\mathrm{O}$ momento $M$ pode ser calculado como

$$
M=F d
$$

em que $F$ é a força aplicada (N) e $d$ é a distância da extremidade fixa da haste ao centro do corpo de prova $(\mathrm{mm})$.

O momento de inércia $I$ da secção reta transversal do corpo de prova, usado em formas retangulares, é dador por

$$
I=\frac{b d^{3}}{12}
$$

em que $a$ é a altura (mm) e $b$ é a largura (mm) da secção transversal da haste.

\subsection{Funcionamento e montagem do Sistema}

O microcontrolador ESP32 envia um sinal de excitação para o HX711 que ativa a ponte Wheastone-composta por dois strain gages e dois resistores de mesma resistência. O HX711, por sua vez, amplifica o sinal coletado com ganho de sinal 128 vezes maior que a saída da ponte. Esse sinal analógico, é convertido para digital digital pelo ADC de 24 bits e, posteriormente, é enviado para o ESP32, que envia os dados via Wi-Fi a um servidor criado em outro ESP32. Por meio da porta serial do ESP32, realiza-se a aquisição de dados em um computador. O sistema completo é composto por 8 resistores e 4 potenciômetros de ajuste, 8 extensômetros, 4 HX711 e 2 ESP32 Sparkfun Thing (cliente e servidor), que coletam e enviam os dados de deformação em 4 pontos diferentes em um tambor descascador de toras de madeira. O circuito é apresentado na Figura 8, em forma esquemática, e na Figura 9 como imagem do sistema montado.

A fixação dos extensômetros é mostrada nas Figuras 10 e 11. Na Figura 12, apresenta-se a fixação do sistema de aquisição no tambor.

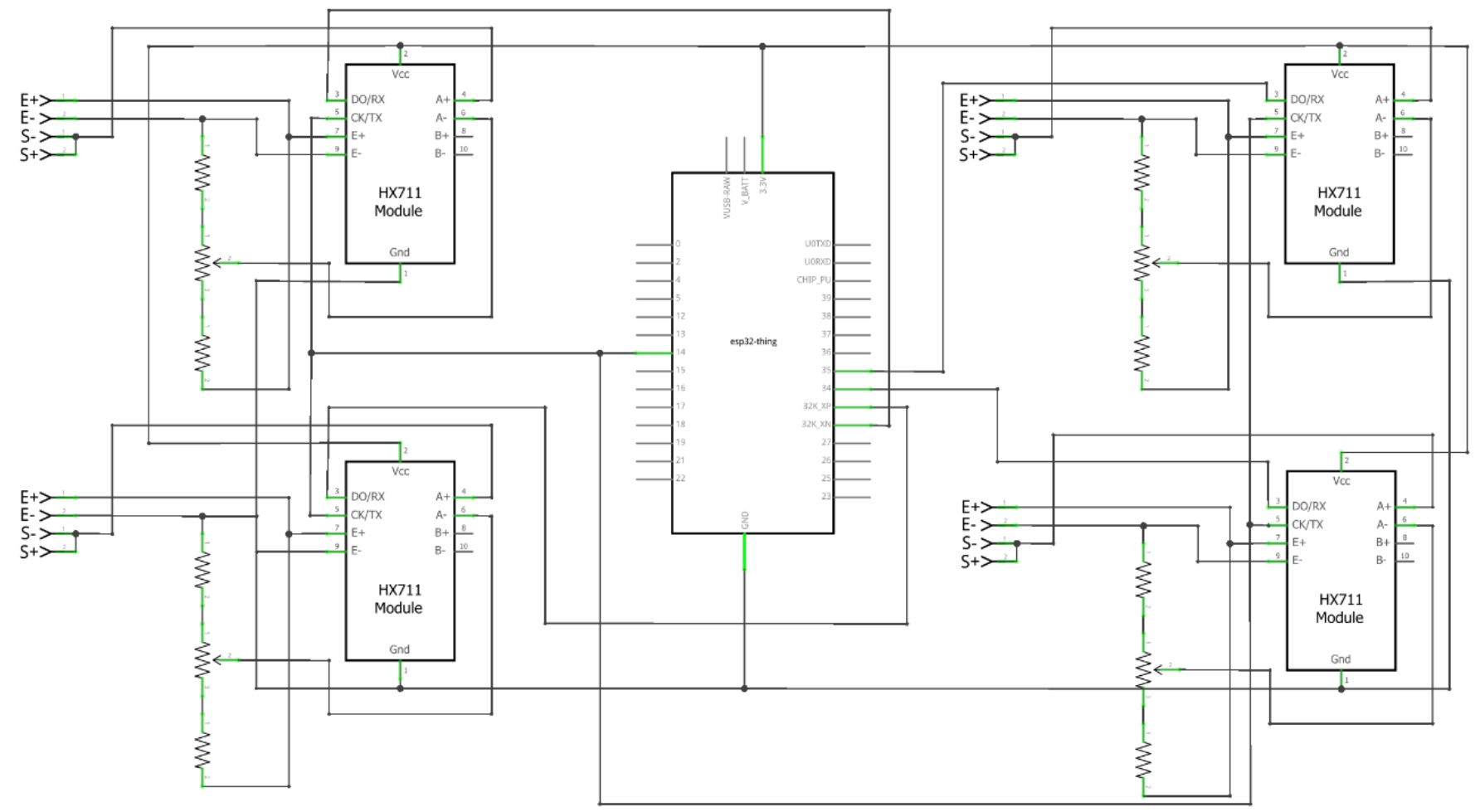

Fig. 8: Diagrama esquemático do sistema de aquisição de dados 


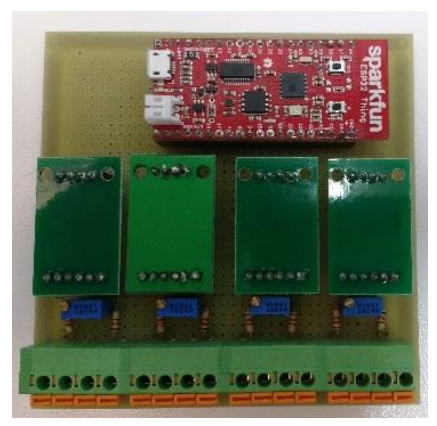

Fig. 9: Placa do sistema de aquisição de dados

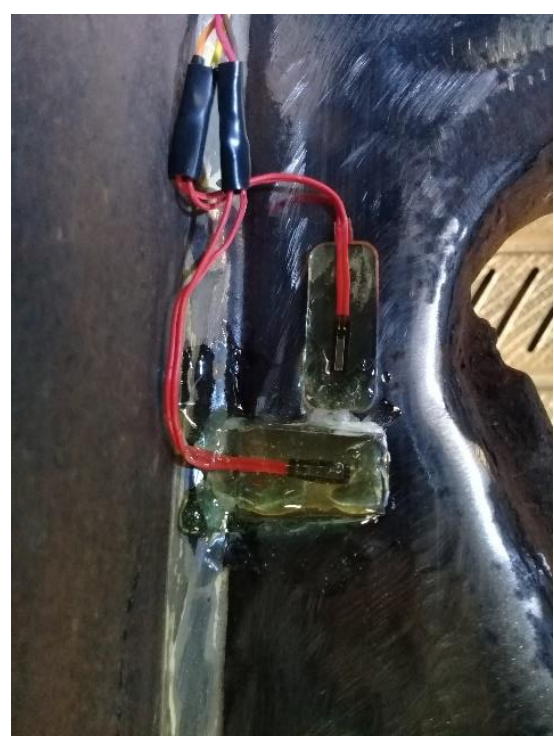

Fig. 10: Pontos de fixação dos extensômetros no tambor descascador de toras.

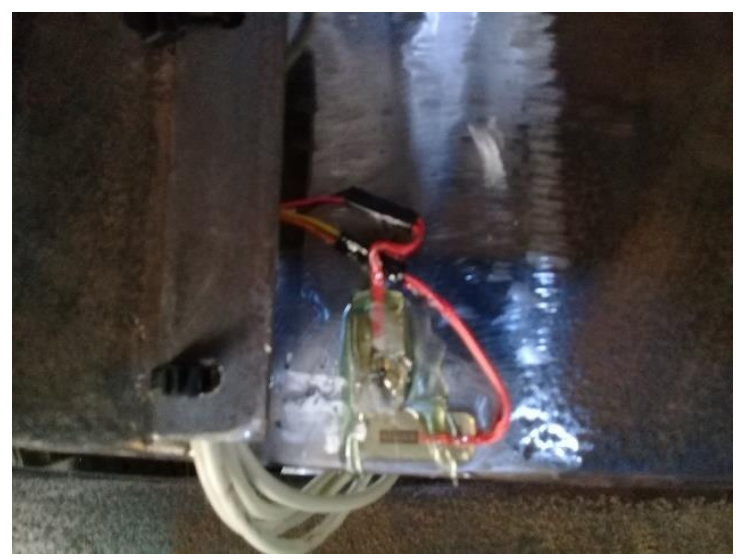

Fig. 11: Pontos de fixação dos extensômetros no tambor descascador de toras.

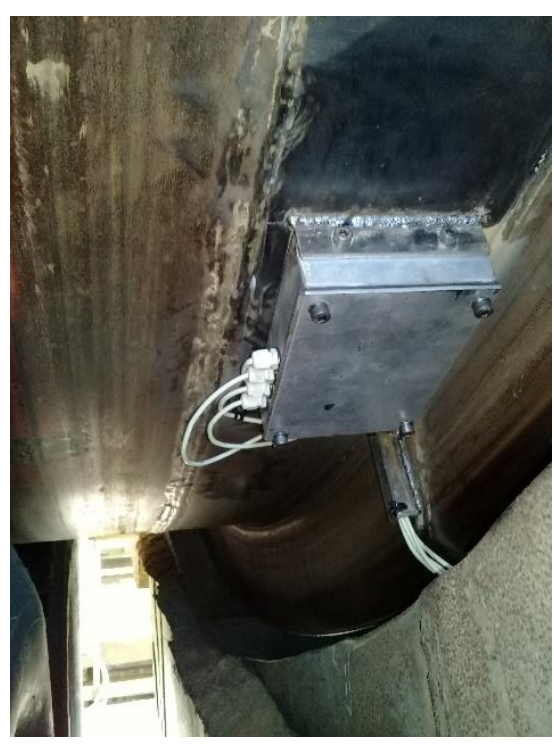

Fig. 12: Ponto de fixação do sistema de aquisição de dados no tambor descascador de toras

\section{RESULTADOS}

Nesta seção são apresentados os resultados do sistema de aquisição de dados na medição dos impactos em um tambor descascador.

\subsection{Calibração do sistema}

Os dados coletados durante o processo de calibração estam apresentados na Tabela 1. Por meio desses dados, ajustou-se a a curva de calibração, apresentada na Figura 13.

Tabela 1. Tabela de dados de calibração

\begin{tabular}{|c|c|c|c|c|c|}
\hline Posição & $\begin{array}{c}\text { Peso } \\
{[\mathbf{N}]}\end{array}$ & $\begin{array}{c}\text { Distância } \\
{[\mathbf{m m}]}\end{array}$ & $\begin{array}{c}\text { Momento } \\
{[\mathbf{N} . m m]}\end{array}$ & $\begin{array}{c}\text { Tensão } \\
{[\mathbf{M P a}]}\end{array}$ & $\begin{array}{c}\text { Resultado } \\
\text { do ADC }\end{array}$ \\
\hline 1 & 2,18 & 100 & 218 & 4,8 & 24849 \\
\hline 2 & 2,18 & 150 & 327 & 7,21 & 36490 \\
\hline 3 & 2,18 & 200 & 436 & 9,61 & 50540 \\
\hline 4 & 2,18 & 250 & 545 & 12,01 & 64121 \\
\hline
\end{tabular}

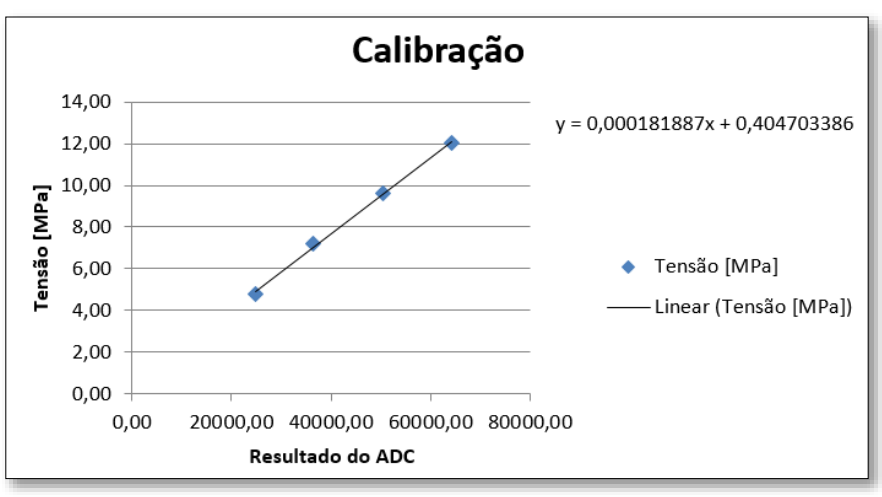

Fig. 13 Gráfico da curva de calibração.

\subsection{Coleta de dados}

Após a instalação do sistema no tambor, os dados foram coletados a $40 \mathrm{~Hz}$, por aproximadamente 30 minutos, gerando 
os dados de cada ponto coletado. Em seguida, os dados foram aplicados à equação de calibração para se obter as medições de cada ponto em tensão (MPa), como mostrados nos gráficos das Figuras 14, 15, 16 e 17.

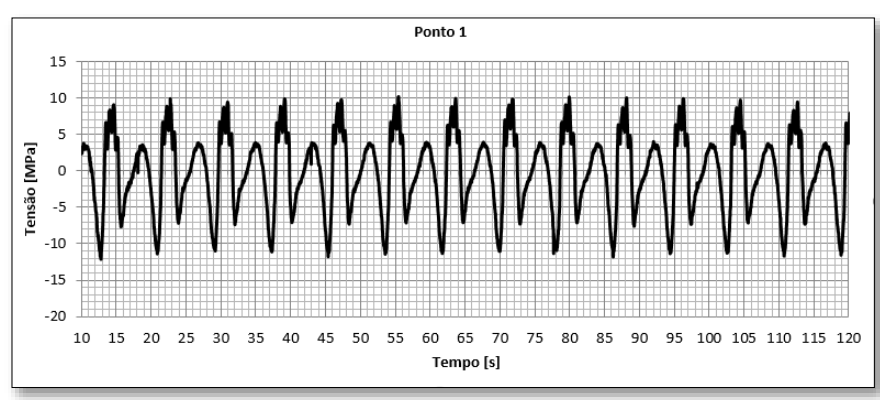

Fig. 14: Medições de tensão no ponto 1.

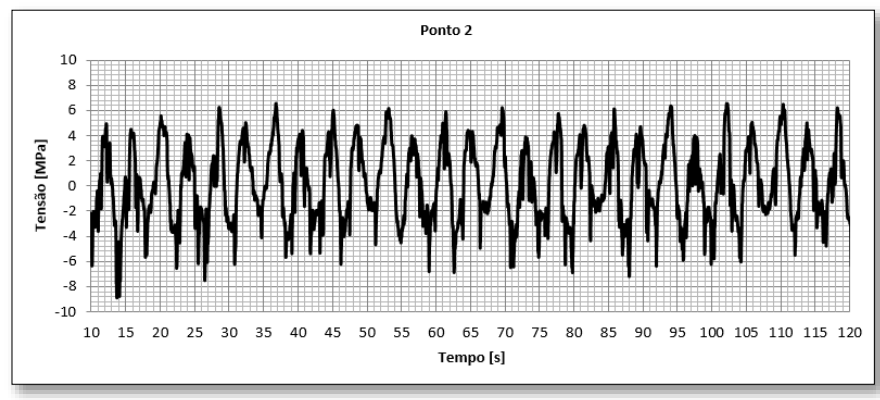

Fig. 15: Medições de tensão no ponto 2.

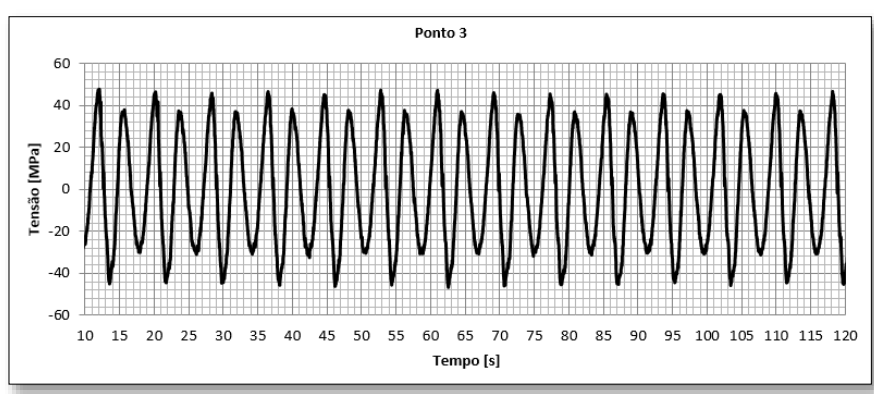

Fig. 16: Medições de tensão no ponto 3.

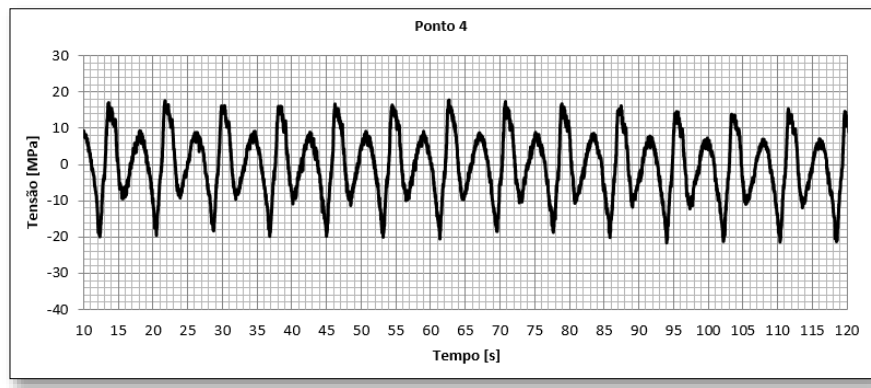

Fig. 17: Medições de tensão no ponto 4.

\section{CONSIDERAÇÕES FINAIS}

Neste trabalho foi apresentado o desenvolvimento de um sistema de aquisição de dados de extensometria aplicado a um tambor descascador de toras de madeira numa fábrica de celulose. Os resultados obtidos são muito importantes para a análise de impacto gerada durante o procedimento com a madeira, podendo sugerir intervenções e/ou manutenção no equipamento.

Os gráficos e dados coletados durante o experimento foram enviados para um estudo de integridade estrutural, em que os dados medidos serão confrontados com os dados simulados em software. A partir desses estudos, reforços mecânicos podem ser sugeridos na nova estrutura do tambor descascador de toras ou na construção de um outro tambor.

\section{AGRADECIMENTOS}

Os autores gostariam de agradecer o apoio da UFOP e do ISQ Brasil para a realização deste trabalho.

\section{REFERÊNCIAS}

Andolfato, R. P., Camacho, J. S., \& Brito, G. D. (2004). Extensometria básica. NEPAE, Ilha Solteira, SP.

Semiconductor, AVIA. (2016). 24-Bit Analog-to-Digital Converter $(A D C)$ for Weigh Scales. Datasheet. AVIA, China.

Guadagnini, P. H., Rocha, F. D., \& Barlette, V. E. (2011). Projeto de um sensor eletrônico baseado em extensometria para medição de força. Latin-American Journal of Physics Education, 5, 753-762.

OMEGA (Brasil). Introdução a sensores de deformação. Disponível em: <http://br.omega.com/prodinfo/sensoresde-deformacao.html>. Acesso em: 19 mar. 2019.

Paulino, H. L. (2011). Aplicação de Strain Gages Rosetas na Análise Experimental de Tensões e Células de Carga e Transdutores de Pressão e Torque. Strain Gage Assessoria em Extensometria Ltda.

Silva, A. L., Varanis, M., Mereles, A. G., Oliveira, C., \& Balthazar, J. M. (2019). A study of strain and deformation measurement using the Arduino microcontroller and strain gauges devices. Revista Brasileira de Ensino de Física, 41(3).

Sparkfun. (2019). ESP32 Thing Hookup Guide. Disponível em: <https://learn.sparkfun.com/tutorials/esp32-thinghookup-guide>. Acesso em: 20 mar. 2019.

National Instruments Corporation. (2019). O que é aquisição de dados?. Disponível em: <http://www.ni.com/dataacquisition/what-is/pt/>. Acesso em: 19 mar. 2019. 